\title{
Polymorphism of Chiral Thioester Studied by Dielectric Spectroscopy
}

\author{
A.I. Wawrzyniak ${ }^{a}$, J. Chruściel ${ }^{b}$, R. Douali ${ }^{c}$, F. Dubois ${ }^{c}$, J.-M. Leblond $^{c}$, \\ Ch. Legrand ${ }^{c}$, D.M. Ossowska-Chruściel ${ }^{b}$, M. MarzeC $^{a, *}$, P. Ropa $^{c}$, J.M. Czerwiec $^{a}$ \\ AND S. WRÓBEL $^{a}$ \\ ${ }^{a}$ Jagiellonian University, Institute of Physics, W.S. Reymonta 4, 30-059 Kraków, Poland \\ ${ }^{b}$ Institute of Chemistry, Siedlce University of Natural Sciences and Humanities, 3-go Maja 54, 08-110 Siedlce, Poland \\ ${ }^{c}$ Université du Littoral Côte d'Opale, 50 rue F. Buisson, B.P. 717, 62228 Calais, France
}

(S)-(+)-4'-[(4-(1-methylheptyloxycarbonylphenyl) thiocarbonylphenyl]-decyloxybenzoate (in short 10.OPOSMH) was studied by complementary methods. The substance shows rich polymorphism. In addition to ferroelectric and antiferroelectric smectic $\mathrm{C}^{*}$ phases it possesses three ferrielectric sub-phases. Phase transitions between the phases were revealed by differential scanning calorimetry and static dielectric permittivity measurements. The aim of this paper was to study phase transitions and differences in dielectric spectra shown by different phases. Collective and molecular dynamics of all phases shown by 10.OPOSMH will be discussed in terms of theoretical models. Dielectric spectra of antiferroelectric phase show two characteristic dielectric relaxation modes: one connected with the molecular process (reorientation around the short axis) and the other originating from fluctuations of antiferroelectric order parameters.

DOI: 10.12693 /APhysPolA.124.933

PACS: 77.84.Nh, 64.70.M-, 77.22.Gm, 77.22.Ej

\section{Introduction}

Since 1988 when antiferroelectric liquid crystals (AFLCs) were synthesized for the first time [1], a lot of experimental [2] and theoretical [3] work has been done to study their dielectric and electrooptic properties. AFLCs have become a very interesting field of research due to their practical applications in science and technology. The prototype of the AFLC compound was MHOPBC. It has been found that AFLC materials $[1,2]$ exhibit not only the anticlinic phase, known as a $\mathrm{SmC}_{\mathrm{A}}^{*}$ phase, but also three other sub-phases [4-6], which were much more difficult to be identified. It is also clear that these sub-phases appear in a phase diagram when the optical purity of the sample increases. If there will be added larger and larger amounts of the enantiomer with opposite handedness, only $\mathrm{SmC}^{*}$ and $\mathrm{SmC}_{\mathrm{A}}^{*}$ phases prevail [1, 2]. Since 1989 many efforts both experimental and theoretical have been made to find out which of the sub-phases are stable. Most of this research was done for AFLCs, which are MHPOBC analogues.

This article reports studies of dielectric properties of a chiral liquid crystal which belongs to a new homologous series of thiobenzoates. Its chemical formula can be written as follows: $\mathrm{C}_{10} \mathrm{H}_{21} \mathrm{O}-\mathrm{C}_{6} \mathrm{H}_{4}-\mathrm{COO}-$ $\mathrm{C}_{6} \mathrm{H}_{4}-\mathrm{COS}-\mathrm{C}_{6} \mathrm{H}_{4}-\mathrm{COO}-\mathrm{CH}\left(\mathrm{CH}_{3}\right) \mathrm{C}_{6} \mathrm{H}_{13}$, abbreviated to $10 . \mathrm{OPOSMH}$. It is interesting that 10.OPOSMH is AFLC material which exhibits all of the predicted basic

*corresponding author; e-mail: monika.marzec@uj.edu.pl sub-phases. 10.OPOSMH was studied by complementary methods, and some of the results were reported earlier in $[4,5]$.

\section{Experimental results and discussion}

\subsection{DSC measurements}

Phase transitions have been investigated by Pyris 1 DSC calorimeter. As found there are many anomalies seen on the thermograms. In addition to a synclinic $\mathrm{SmC}^{*}$ ferroelectric and anticlinic antiferroelectric phase $\mathrm{SmC}_{\mathrm{A}}^{*}$ there are three sub-phases: $\mathrm{SmC}_{\alpha}^{*}, \mathrm{SmC}_{\beta}^{*}, \mathrm{SmC}_{\gamma}^{*}$.

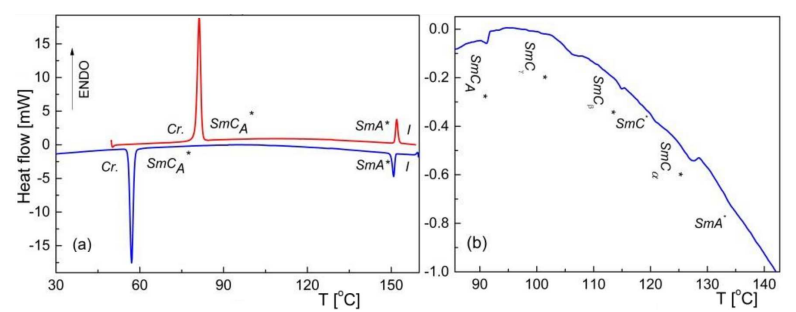

Fig. 1. Calorimetric results obtained during heating and cooling of 10.OPOSMH sample (left) and small anomalies between sub-phases present in the cooling mode (right).

Differential scanning calorimetry (DSC) curves were recorded during heating and cooling of the sample in the whole temperature range. As one can see on the heating curve there are two anomalies corresponding to the melting point (at temperature of $79.3^{\circ} \mathrm{C}$ ) and to the clearing point (at $150.4^{\circ} \mathrm{C}$ ). The zoomed picture of the cooling 
DSC curve (Fig. 1b) reveals some additional anomalies in the temperature range between $100^{\circ} \mathrm{C}$ and $135^{\circ} \mathrm{C}$. The phases appearing between the paraelectric $\mathrm{Sm}^{*}$ and antiferroelectric $\mathrm{SmC}_{\mathrm{A}}^{*}$ are recognized as follows: $\mathrm{SmC}_{\alpha}^{*}$, $\mathrm{SmC}^{*}, \mathrm{SmC}_{\beta}^{*}, \mathrm{SmC}_{\gamma}^{*}$, respectively. Based on $\mathrm{DSC}$ and a static electric permittivity measurements, one can determine the phase sequence. The phase sequence obtained during cooling the sample is as follows:

$$
\begin{aligned}
& \mathrm{I} 149.7^{\circ} \mathrm{C} \mathrm{Sm}{ }^{*} 126^{\circ} \mathrm{C} \mathrm{SmC}_{\alpha}^{*} 124.9^{\circ} \mathrm{C} \mathrm{SmC}^{*} 114.5^{\circ} \mathrm{C} \\
& \mathrm{SmC}_{\beta}^{*} 111^{\circ} \mathrm{C} \mathrm{SmC}_{\gamma}^{*} 109.6{ }^{\circ} \mathrm{SmC}_{\mathrm{A}}^{*} 59.3^{\circ} \mathrm{C} \mathrm{Cr} .
\end{aligned}
$$

\subsection{Spontaneous polarization measurements}

To find out the temperature of the synclinic $\mathrm{SmC}^{*}$ ferroelectric phase spontaneous polarization has been measured. For those studies ITO cells WAT were employed. Spontaneous polarization measurements have been performed by the reversal current method. The experimental setup consists of Agilent 3310 waveform generator, AFLC electronics F20 DI amplifier and digital osciloscope gilent DSO6102A.

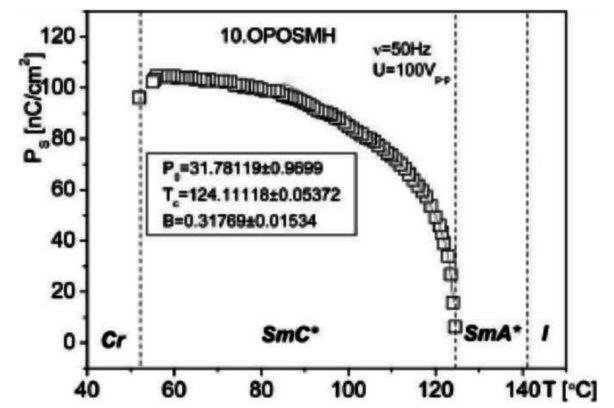

Fig. 2. Spontaneous polarization of 10.OPOSMH vs. temperature $\left(\nu=50 \mathrm{~Hz}, U=100 V_{p-p}\right)$.

As seen in Fig. 2 the spontaneous polarization has intermediate values between highly polar and those exhibiting low macroscopic polarity, which favors a rich phase polymorphism. Solid line is a fit of the mean-field function

$$
P_{\mathrm{S}}(T)=P_{0}\left(T-T_{\mathrm{C}}\right)^{\beta},
$$

where $T_{\mathrm{C}}$ is the critical temperature (the Curie temperature) and $\beta$ is the exponent, which according to the theory for the second order transition is equal to 0.5 . In the case of the substance studied the value of $0.317 \pm 0.015$ is closer to that foreseen for tri-critical point $(\beta=0.25)$. The Curie temperature of 10.OPOSMH is $T_{\mathrm{C}}=124.11 \pm$ $0.05^{\circ} \mathrm{C}$.

\subsection{Dielectric studies}

Dielectric spectroscopy measurements were performed at ULCO (Université du Littoral Côte d'Opale) in Calais in the frequency range between $30 \mathrm{~Hz}$ and $1 \mathrm{MHz}$ using HP4284 Impedance Material nalyzer, which incorporates 200 frequency channels. This model offers measuring fields between $100 \mathrm{mV}$ and $10 \mathrm{~V}$. AWAT HG electro-optic cell $(d=9 \mu \mathrm{m})$ was filled with the sample in its isotropic phase by a capillary effect.

Figure 3 presents temperature dependence of dielectric permittivity of 10.OPOSMH for three selected frequencies. As seen, the substance studied exhibits para-, ferro-, and antiferroelectric phases and three $\mathrm{SmC}^{*}$ sub-phases.

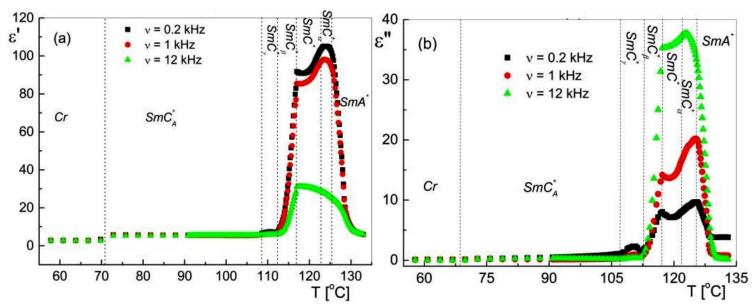

Fig. 3. Dielectric permittivities measured on cooling at different frequencies vs. temperature showing rich polymorphism of 10.OPOSMH compound (a) and dielectric loss vs. temperature (b).

The static dielectric permittivity, measured at the lowest frequency of $0.2 \mathrm{kHz}$, shows anomalies connected with phase transitions. The $\mathrm{SmC}_{\alpha}^{*}$ sub-phase shows an enhanced dielectric permittivity, which distinctly decreases upon the $\mathrm{SmC}_{\alpha}^{*}-\mathrm{SmC}^{*}$ transition, and finally drastically is reduced at the transition (via $\mathrm{SmC}_{\beta}^{*}$ and $\mathrm{SmC}_{\gamma}^{*}$ sub-phases) to the antiferroelectric phase $\mathrm{SmC}_{\mathrm{A}}^{*}$. At the $\mathrm{SmC}_{\mathrm{A}}^{*}-\mathrm{Cr}$ transition there is a small change of the static dielectric permittivity, which means that the reorientational motions of molecules in the antiferroelectric phases are very limited.

To explain frequency dependences of both components of the complex dielectric permittivity measured for different phases one can use the following formula [6]:

$$
\begin{aligned}
& \varepsilon^{*}(\omega)=\varepsilon^{\prime}(\omega)-\mathrm{i} \varepsilon^{\prime \prime}(\omega) \\
& =\varepsilon(\infty)+\sum_{j} \frac{\varepsilon_{j}(0)-\varepsilon_{j}(\infty)}{1+\left(\mathrm{i} \omega \tau_{j}\right)^{1-\alpha_{j}}}-\frac{\mathrm{i} \sigma}{\varepsilon_{0} \omega^{M}},
\end{aligned}
$$

where $\omega$ is a circular frequency of the measuring electric field, $\varepsilon_{j}(0)$ and $\varepsilon_{j}(\infty)$ are, respectively, the static and the high frequency limit of the dielectric permittivity for the $j$-th relaxation process, $\tau_{j}$ is the dielectric relaxation time, $\varepsilon_{0}$ - an electric permittivity of the free space, $\sigma$ - static conductivity, $\alpha_{j}$ and $M$ are fitting parameters introduced to explain deviations from the Debye dielectric relaxation and pure ionic conductivity contribution, respectively.

As seen in Fig. 4, the dielectric studies done in the whole temperature range without bias field disclosed one relaxation process in the $\mathrm{Sm}^{*}, \mathrm{SmC}_{\alpha}^{*}, \mathrm{SmC}^{*}$, and $\mathrm{SmC}_{\beta}^{*}$ phases, whereas in the $\mathrm{SmC}_{\gamma}^{*}$ and $\mathrm{SmC}_{\mathrm{A}}^{*}$ two dielectric processes have been obtained. As found, in all phases studied the difference between $\varepsilon(\infty)$ and refractive index squared is close to 0.5 which shows that there is a fast dielectric relaxation process (reorientation around the long molecular axis) falling in the microwave frequency range. 

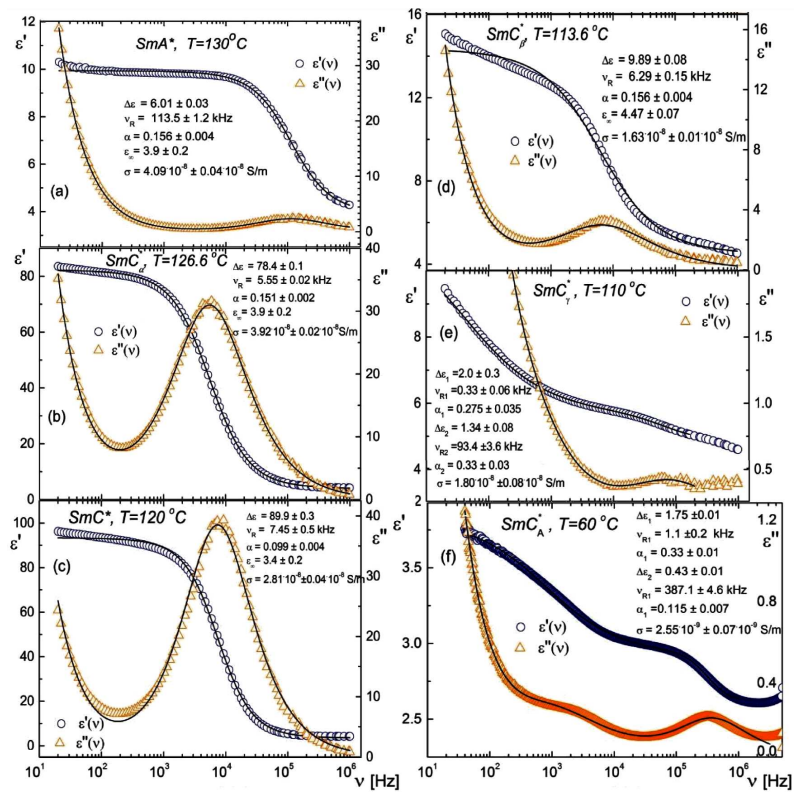

Fig. 4. Dielectric spectra with fitting parameters obtained in different smectic phases: paraelectric $\mathrm{Sm}^{*}$ (a), $\mathrm{SmC}_{\alpha}^{*}$ (b), ferroelectric $\mathrm{SmC}^{*}$ (c), $\mathrm{SmC}_{\beta}^{*}$ (d), ferrielectric $\mathrm{SmC}_{\gamma}^{*}$ (e), and antiferroelectric $\mathrm{SmC}_{\mathrm{A}}^{*}$ (f) of 10.OPOSMH. Solid lines were computed using Eq. (2).

As an example in Fig. 4b one can see a complex spectrum of the $\mathrm{SmC}_{\alpha}^{*}$ sub-phase with the fitting parameters showing that the dielectric spectrum of this phase is not a Debye-type. On the other hand, the $\mathrm{SmC}^{*}$ and $\mathrm{Sm}^{*}$ phases (Fig. 4a and c) display the Debye-type dielectric spectra of the Goldstone and soft mode, respectively.

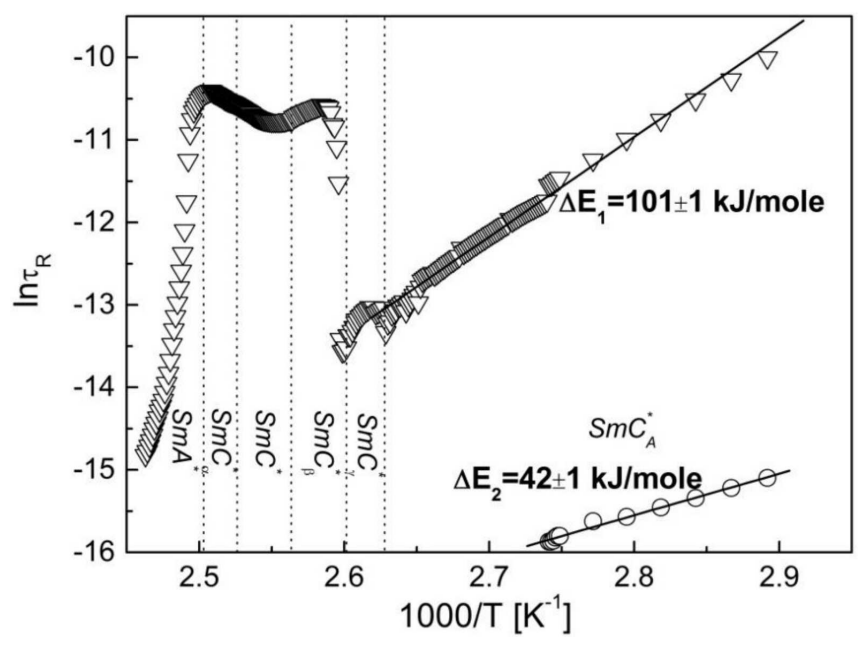

Fig. 5. Arrhenius plot for the relaxation times measured for different phases of 10.OPOSMH compound.

For better interpretation of dielectric processes, the Arrhenius plot (Fig. 5) was done to compute the activation energies. In the $\mathrm{Sm}^{*}$ phase there is a characteristic soft mode (SM). In the $\mathrm{SmC}_{\alpha}^{*}$ and $\mathrm{SmC}^{*}$ phases the low fre- quency Goldstone mode (GM) shows up. The SM and GM are non-Arrhenius relaxation processes. The collective process in the $\mathrm{SmC}_{\beta}^{*}$ phase (AFM1) can be connected with fluctuation of the tilt angle. Similar behavior was observed in [7]. In the $\mathrm{SmC}_{\gamma}^{*}$ and $\mathrm{SmC}_{\mathrm{A}}^{*}$ two dielectric processes have been revealed, but the low frequency mode in the $\mathrm{SmC}_{\gamma}^{*}$ is very weak and difficult to observe in the whole temperature range of this phase. As seen, only in the $\mathrm{SmC}_{\mathrm{A}}^{*}$ phase the dielectric relaxation processes have an activation-like character following the $\mathrm{Ar}$ rhenius behavior. The low frequency process (AFM2) may originate from the tumbling of molecules around their short molecular axes with activation energy of $c a$. $101 \mathrm{~kJ} / \mathrm{mol}$, which is also observed in the antiferroelectric $\mathrm{SmC}_{\mathrm{A}}^{*}$ phase for other compounds [2, 7-12]. For the antiferroelectric phase there is another complex collective process (AFM2) with activation energy of $c a .42 \mathrm{~kJ} / \mathrm{mol}$, which is due to fluctuations of the tilt order parameters in adjacent layers. It is always difficult to interpret the relaxation processes occurring in the $\mathrm{SmC}_{\mathrm{A}}^{*}$ phase, but similar behavior was also discussed in [7-9]. The high frequency limit of the dielectric permittivity is close to 4 and it contains contributions from the reorientation around the long molecular axis and/or intra-molecular reorientations. Our results agree qualitatively with the theoretical predictions based on the assumption that in the antiferroelectric phase in-phase and anti-phase fluctuations of the molecules are dielectrically active giving two dielectric modes [2,3]. However, the theory predicts that the low frequency dielectric mode is present only under the bias field. In the case of the substance studied it is present without bias field. In our previous studies of this compound performed under different experimental conditions it was shown that the antiferroelectric phase also exhibit two dielectrically active modes whereas in the $\mathrm{SmC}_{\gamma}^{*}$ sub-phase three separate dielectric processes reveal [5].

\section{Conclusions and perspectives}

I. It is presented that each phase shows its characteristic dielectric spectrum. The spectra have symmetric distribution of the dielectric relaxation times. It is sufficient to use Cole--Cole function with a conductivity term (Eq. (2)).

II. Dielectric spectra of the antiferroelectric phase show two characteristic dielectric relaxation modes: one connected with the molecular process (flip-flop jumps) and the other coming from fluctuations of antiferroelectric order parameter.

III. Relaxation processes found for the paraelectric, ferro- and ferrielectric phases are non-Arrhenius ones, whereas the antiferroelectric phase shows two Arrhenius dielectric relaxation processes. 


\section{Acknowledgments}

The authors would like to thank the State Committee for Scientific Research (KBN) for partial financial support in scope of the grant No. NN 202076435.

The research was carried out with the equipment purchased thanks to the financial support of the European Regional Development Fund in the framework of the Polish Innovation Economy Operational Program (contract no. POIG.02.01.00-12-023/08).

\section{References}

[1] A.D.L. Chandani, E. Górecka, Y. Ouchi, H. Takezoe, A. Fukuda, Jpn. J. Appl. Phys. 28, L1265 (1989).

[2] M. Fukui, H. Orihara, Y. Yamada, N. Yamamoto, Y. Ishibashi, Jpn. J. Appl. Phys. 28, L849 (1989).

[3] E. Górecka, D. Pociecha, M. Čepič, B. Žekš, R. Dąbrowski, Phys. Rev. E 65, 061703 (2002).

[4] D.M. Ossowska-Chruściel, Phase Transit. 80, 757 (2007).

[5] M. Marzec, M. Bohdan, M.D. Ossowska-Chruściel, J. Chruściel, S. Wróbel, Mol. Cryst. Liq. Cryst. 540, 227 (2011).
[6] H. Kresse, in: Relaxation Phenomena, Eds. W. Haase, S. Wróbel, Springer-Verlag, Berlin 2003, Ch. 5.7.

[7] A. Mikułko, M. Marzec, R. Douali, Ch. Legrand, S. Wróbel, R. Dąbrowski, Mol. Cryst. Liq. Cryst. 439, 2135 (2005).

[8] A. Mikułko, Ph. D. Thesis, Jagiellonian University, Kraków 2006.

[9] S. Wróbel, A. Fąfara, M. Marzec, W. Haase, R. Dąbrowski, W. Drzewiński, B. Gestblom, M. Makrenek, Phase Transit. 80, 791 (2007).

[10] L.A. Parry-Jones, S.J. Elston, Phys. Rev. E 63 , 050701 (2001).

[11] L.A. Parry-Jones, S.J. Elston, J. Appl. Phys. 92, 449 (2002).

[12] M. Marzec, A. Mikułko, S. Wróbel, W. Haase, in: Dielectric Properties of Liquid Crystals, Eds. Z. Galewski, L. Sobczyk, Transworld Research Network, Kerala 2007, Par. 4, p. 83. 